\title{
CRECIMIENTO Y CALIDAD DEL TALLO FLORAL DE FREESIA X HYBRIDA EN HIDROPONÍA
}

\author{
GROWTH AND STEM QUALITY OF FREESIA X HYBRIDA UNDER HYDROPONICS
}

\author{
Érika Miranda-Villagómez ${ }^{1}$, J. Alfredo Carrillo-Salazar ${ }^{1 \star}$, María-de-las-Nieves Rodríguez-Mendoza ${ }^{1}$, \\ M. Teresa Colinas-León ${ }^{2}$, Manuel Livera-Muñoz ${ }^{1}$ y E. Araceli Gaytán-Acuña ${ }^{1}$
}

\begin{abstract}
${ }^{1}$ Recursos Genéticos y Productividad-Fisiología Vegetal, Campus Montecillo, Colegio de Posgraduados. Km 36.5 Carretera México-Texcoco. 56230, Montecillo, Texcoco, Edo. de México. Tel. 01 (595) 952-0200 Ext. 1593. ${ }^{2}$ Departamento de Fitotecnia, Universidad Autónoma Chapingo, Km 38.5 Carretera México-Texcoco, Chapingo, Texcoco, Edo. de México. Tel. 01 (595) 952-1500 Ext. 5224.
\end{abstract}

*Autor para correspondencia (asalazar@colpos.mx)

\section{RESUMEN}

Freesia (Freesia $x$ hybrida) tiene alto potencial como flor de corte pero es poco comercializada en México; tolera bajas temperaturas, produce amplia diversidad de colores y tiene un aroma agradable. En esta investigación se evaluó el efecto de la concentración de la solución nutritiva de Steiner en el crecimiento y calidad del tallo floral de Freesia $x$ hybrida cv. 'Patio' generada a partir de cormos de $2.5 \mathrm{~cm}$ de diámetro, crecida bajo hidroponía y sombreada con malla roja a $85 \%$, durante el invierno en el Valle de México. Los tratamientos fueron la combinación factorial de cuatro concentraciones de solución nutritiva (SN) Steiner $(25,50,75$ y $100 \%)$ y dos fechas de siembra (FS) (22 de septiembre y 18 de octubre de 2010), en un diseño completamente al azar. Al inicio de la floración, con la solución Steiner a $100 \%$ se obtuvieron plantas con menores valores de altura de planta $(49 \mathrm{~cm})$, longitud del tallo floral $(37 \mathrm{~cm})$, área foliar por planta $\left(170 \mathrm{~cm}^{2}\right)$, lecturas SPAD (62), longitud de la espiga $(8.5 \mathrm{~cm})$, número de flores por espiga (8), grosor del tallo $(3.6 \mathrm{~mm})$, biomasa seca de tallo, hoja y total, mientras que con la concentración de $\mathbf{2 5} \%$ se obtuvieron los mayores valores de altura de planta $(59.4 \mathrm{~cm})$, lecturas SPAD (71.7), longitud del tallo floral (61 $\mathrm{cm})$, longitud de la espiga $(11 \mathrm{~cm})$, número de flores por espiga (12), grosor del tallo $(5.0 \mathrm{~mm})$ y de vida de florero $(12 \mathrm{~d})$. En el número de hojas por planta y en la biomasa seca de raíz no hubo diferencias entre tratamientos. La eficiencia cuántica máxima del fotosistema II $(\mathrm{Fm} / \mathrm{Fv})$ fue igual entre tratamientos $(P>0.05)$ en ambas fechas de medición, con un promedio de 0.81 .

Palabras clave: Freesia $x$ hybrida, solución nutritiva, crecimiento, calidad del tallo floral.

\section{SUMMARY}

Freesia (Freesia $x$ hybrida) is a high-potential cut-flower, but it is almost unknown in México. It tolerates low temperatures and forms flowers in a wide range of colors with a delicate scent. This research evaluated the effect of Steiner solution concentration on growth and flower stem quality of Freesia $x$ hybrida cv. 'Patio' grown in the Valley of México. Plants were derived from 2.5 diameter corms, under hydroponics and $85 \%$, red shade netting during the Winter season. The experimental design was a factorial combination of four Steiner solution concentrations $(25,50,75$ and $100 \%)$ and two planting dates (September 22nd and October 18th. 2010), under a completely random design. The $100 \%$ Steiner solution (SN) produced the lowest values in plant height $(49 \mathrm{~cm})$, floral stem length $(37 \mathrm{~cm})$, leaf area per plant $\left(170 \mathrm{~cm}^{2}\right)$, SPAD readings $(62)$, spike length $(8.5 \mathrm{~cm})$, number of flowers per stem (8), stem thickness $(3.6 \mathrm{~mm})$, and in stem, leaf and total dry weight; while the $25 \% \mathrm{SN}$ produced the highest values in plant height $(59.4 \mathrm{~cm})$,
SPAD readings $(71.7)$, floral stem length $(61 \mathrm{~cm})$, spike length $(11 \mathrm{~cm})$, number of flowers per stem (12), stem thickness $(5.0 \mathrm{~mm})$ and flower vessel life (12 d). Neither the number of leaves per plant nor the root dry weight were different among treatments. The maximum quantum efficiency of photosystem II $(\mathrm{Fm} / \mathrm{Fv})$ was similar in all treatments $(\mathbf{P}>$ 0.05), in both planting dates, with an average of 0.81 .

Index words: Freesia $x$ hybrida, nutrient solution, plant growth, floral stem quality.

\section{INTRODUCCIÓN}

El cultivo de las flores es uno de los agronegocios más rentables en el mundo. Desde la década de los ochentas, Holanda ocupa uno de los primeros lugares como país productor, actividad que ha formado parte de su cultura por décadas. Otros países, como Estados Unidos, Japón e Italia, tienen actualmente altos ingresos por la producción de flores de corte y maceta. Por su parte, países como India, China, Kenia, Ecuador, Colombia y México tienen alto potencial económico, climático, geográfico y comercial para crecer en el ramo (ASERCA, 2006).

Existen especies florícolas cultivadas que tienen alto potencial como flor de corte pero que son poco comercializadas en México. Tal es el caso de freesia (Freesia $x$ hybrida), especie originaria de Sudáfrica (Wang, 2007). Las variedades disponibles en el mercado corresponden a híbridos (Freesia $x$ hybrida) obtenidos mediante cruzamientos entre las especies Freesia alba, F. refracta, F. corymbosa y F. leichtlinnii (Dole y Wilkins, 1999). Freesia $x$ hybrida tiene mayores valores en longitud de vara, tamaño de la flor y resistencia a plagas y enfermedades (Rees, 1992). Esta especie tiene potencial en México porque tolera bajas temperaturas y puede producirse durante el invierno, así como por su aroma y diversidad de colores, aspectos poco explotados en el mercado nacional de flores que pueden ser un atractivo para su consumo. En México la principal demanda de flor de freesia es el 2 de febrero, demanda que se satisface con genotipos quizá introducidos al país después de la conquista 
de México y cultivados de una forma rústica y tradicional, sin conocer con certeza la especie explotada y sin estadísticas de producción para validar su cultivo a nivel comercial en el país.

Diversos autores han evaluado el crecimiento, desarrollo y calidad de la flor de freesia en función de la nutrición, y han encontrado influencia del nitrógeno en el tamaño de hoja, color de la hoja, número de hojas, longitud del tallo floral, tamaño del cormo, crecimiento vegetativo y el tiempo en aparición del tallo floral. Por su parte, el fósforo causa cambios en el tamaño de la hoja, el tiempo en aparición del tallo floral, tiempo de vida de la espiga floral y el peso del cormo (Thomas et al., 1998; Kidsadawanich y Ruamrungsri, 2005; Ruamrungsri et al., 2011; Khan et al., 2012). En la mayoría de las investigaciones se aplicaron fertilizantes comerciales, tanto en el cultivo en suelo como en mezclas de materiales orgánicos e inorgánicos.

Kidsadawanich y Ruamrungsri (2005) y Ruamrungsri et al. (2011) usaron sustratos y solución nutritiva para cultivar esta especie bajo hidroponía. La hidroponía permite desarrollar plantas en soluciones nutritivas (SN), con o sin el uso de un medio artificial (arena, grava, lana de roca, vermiculita, entre otros) para proveer soporte mecánico a la planta (Lara, 1999). La SN consiste en agua con oxígeno y los nutrimentos esenciales en forma iónica (Steiner, 1961). La cantidad de nutrimentos que las plantas requieren depende de la especie, variedad, etapa fenológica y condiciones ambientales (Carpena et al., 1987). La SN de Steiner es una de las más utilizadas en cultivos hidropónicos, ya que sus propiedades (balance entre aniones y cationes, conductividad eléctrica y potencial osmótico) permiten un buen desarrollo de la planta.

El objetivo de esta investigación fue evaluar el efecto de la solución nutritiva de Steiner en el crecimiento y calidad del tallo floral de Freesia $x$ hybrida cultivada bajo un sistema hidropónico y sombreado con malla roja a $85 \%$, durante el invierno en el Valle de México.

\section{MATERIALES Y MÉTODOS}

El estudio se llevó a cabo en dos fechas de siembra (22 de septiembre y 18 de octubre de 2010), en Texcoco, México, a $2250 \mathrm{~m}$ de altitud, $19^{\circ} 29^{\prime} \mathrm{LN}$ y $98^{\circ} 54^{\prime} \mathrm{LO}$, con plantas crecidas bajo malla sombra de color rojo (ChromatiNet Roja Polysack ${ }^{\circledR}$, que transmiten $15 \%$ de la radiación solar). Esta malla tiene una relación entre rojo $(680 \mathrm{~nm})$ y rojo lejano $(740 \mathrm{~nm})$ (R:RL) de 0.82 , medida con un radiómetro multiespectral (MSR, por sus siglas en inglés) marca CROPSCAN ${ }^{\circledR}$ (Cropscan Inc. 2001). Esta malla es usada para producir plantas con hojas más grandes, tallos más largos y anchos, y en general con mayor volumen de follaje.
La irradiancia promedio máxima sobre el follaje fue de $45.19 \mathrm{~W} \mathrm{~m}^{-2} \mathrm{~s}^{-1}$, y fuera de la malla fue $295.33 \mathrm{~W} \mathrm{~m}^{-2} \mathrm{~s}^{-1}$; ésta fue medida con un radiómetro lineal modelo LI-191SE (LI-COR Inc.; Lincoln, Nebraska, USA). La temperatura promedio del aire en el sitio experimental fue $12.3^{\circ} \mathrm{C}$, con máxima de $29.1{ }^{\circ} \mathrm{C}$ y mínima de $-5.6{ }^{\circ} \mathrm{C}$, y fue medida con un sensor ("data logger") modelo H08-003-02 Hobo ${ }^{\circledR}$ (ONSET Inc.).

Las plantas se obtuvieron a partir de cormos de Freesia $x$ hybrida cv. 'Patio' de $2.5 \mathrm{~cm}$ de diámetro. Esta variedad produce flores amarillas, rosadas, rojas, naranja y blancas. Previo a la plantación, los cormos se sumergieron por $15 \mathrm{~min}$ en una solución antifúngica (Captan ${ }^{\circledR} 0.8 \mathrm{~g} \mathrm{~L}^{-1}$ y Benlate ${ }^{\circledR}$ $\left.0.40 \mathrm{~g} \mathrm{~L}^{-1}\right)$. La siembra se hizo en bolsas de plástico negro de $15 \mathrm{x} 25 \mathrm{~cm}$ rellenadas con tezontle (Ø $5 \mathrm{~mm})$ como sustrato, $\mathrm{y}$ se plantaron cinco cormos en cada bolsa.

Los tratamientos consistieron de una combinación factorial de cuatro concentraciones de la solución nutritiva de Steiner 25, 50, 75 y $100 \%\left(\mathrm{NO}_{3}^{-}, 12\right.$ meq; $\mathrm{H}_{2} \mathrm{PO}_{4}^{-}, 1$ meq; $\mathrm{SO}_{4}^{2-}, 7$ meq; $\mathrm{K}^{+}, 7$ meq; $\mathrm{Ca}^{2+}, 9$ meq; $\mathrm{Mg}^{2+}, 7$ meq; para la solución a $100 \%$ ), y dos fechas de siembra F1 (22 de septiembre) y F2 (18 de octubre). La solución se preparó con agua de la llave y se aplicó en riego por goteo, cinco veces al día (5 min cada riego). En cada solución se ajustó el pH a 6 , y la conductividad eléctrica (CE) se midió enseguida con un potenciómetro HI 98129 Hanna ${ }^{\circledR}$ (Hanna Instruments Inc., Woonsocket, Rhode Island, USA). La CE fue 1.1, 1.6, 2.1, y $2.6 \mathrm{dS} \mathrm{m}^{-1}$ para las concentraciones de 25, 50, 75 y 100 $\%$ de la SN. Los ocho tratamientos se distribuyeron en un diseño completamente al azar con 25 repeticiones, en donde cada planta fue una repetición.

Durante el desarrollo del cultivo se hicieron tres muestreos: a la cuarta hoja recientemente madura ( $30 \mathrm{~d}$ después de plantación, DDP), séptima hoja recientemente madura (58 DDP), y flores abiertas o inicio de la floración (145 DDP), con cinco repeticiones por tratamiento y muestreo. En las plantas muestreadas se midió: área foliar (AF) con un integrador LI-3100® (LI-COR, Inc., Lincoln, NE, USA); altura de planta, con una cinta métrica desde la base hasta el ápice de la hoja más joven; número de hojas por planta; y biomasa seca (g) de cada órgano. Las muestras se secaron en una estufa de aire forzado a $70{ }^{\circ} \mathrm{C}$ durante $72 \mathrm{~h}$, y se pesaron en una balanza electrónica modelo 1474 (Sartorius AG®, Göttingen, Deutschland) con precisión de $0.1 \mathrm{~g}$.

En dos fechas ( 13 de diciembre y 25 de enero) se midió la fluorescencia inducida con un aparato Handy PEA (Hansatech Instruments Ltd., King's Lynn, Norfolk, UK) en cinco hojas de plantas diferentes; cada hoja fue una repetición por tratamiento. Se midió el nivel de fluorescencia cuando el 
aceptor de electrones en la plastoquinona (Qa) está completamente oxidado (F0), y cuando Qa está completamente reducido $(\mathrm{Fm})$; la fluorescencia variable $(\mathrm{Fv})$, y la eficiencia quántica máxima del fotosistema II (Fv/Fm) (Hansatech Instruments Ltd., 2006). El índice de verdor fue medido con un aparato SPAD 502 (Minolta Co. Ltd., Osaka, Japan) en tres hojas expandidas en la parte media de la hoja de cinco plantas por tratamiento, como propuesieron Bullock y Anderson (1998).

La cosecha de los tallos florales se hizo cuando la primera flor mostró su color. En esa etapa se midió la longitud y grosor del tallo, con una cinta métrica y un vernier digital modelo CALDI-6MP (TRUPER ${ }^{\circledR}$, China), así como la longitud y número de flores de la espiga. En 10 tallos florales por tratamiento se evaluó la vida en florero mediante: el peso de la biomasa hidratada (cada 2 d) en cada tallo floral; el registro diario del número de flores abiertas; y días a senescencia.

Los datos de las variables se sometieron a un análisis de varianza y a comparaciones de medias con la prueba de Tukey $(\alpha=0.05)$, mediante el programa estadístico SAS versión 9.2 (SAS Institute, 2010). Con el programa Curve Expert ${ }^{\circledR}$ ver. 1.2.2 (http://www.curveexpert.net/) se encontró un modelo de buen ajuste estadístico $\left(\mathrm{R}^{2}\right)$ para modelar el comportamiento de la longitud del tallo floral.

\section{RESULTADOS}

\section{Altura de planta}

En el primer muestreo hubo diferencias significativas entre las soluciones nutritivas $(P \leq 0.05)$, en el segundo muestreo no hubo efecto de concentraciones ni de fechas de siembra $(P>0.05)$, y en el tercer muestreo hubo efecto significativo de fechas de siembra y de concentraciones de la solución nutritiva $(\mathrm{P} \leq 0.05)$. Las plantas de menor tamaño en el primero y tercer muestreo fueron las nutridas con solución nutritiva Steiner a $100 \%$, y las más altas correspondieron a la concentración de $25 \%$ (Cuadro 1).

\section{Número de hojas y área foliar}

En el número de hojas no hubo diferencias significativas entre tratamientos $(\mathrm{P}>0.05)$; cada planta formó de 10 a 11 hojas. En área foliar se detectaron diferencias significativas $(\mathrm{P} \leq 0.05)$ entre concentraciones en los tres muestreos, $\mathrm{y}$ también entre fechas en el segundo y tercer muestreo (Cuadro 2). La Solución Steiner a $50 \%$ favoreció al área foliar medida en el primer muestreo con $51.4 \%$ más que las plantas con menor área que correspondieron a la concentración de $100 \%$. En el segundo muestreo las plantas tuvieron 19 \% más área foliar con la solución nutritiva a 75 \% que con el resto de las concentraciones, mientras que en el tercer muestreo la mayor área foliar correspondió a la SN de 50 $\%$ (Cuadro 2).

\section{Biomasa seca de cormo y planta}

En esta variable no hubo diferencias significativas entre fechas de siembra $(\mathrm{P}>0.05)$, pero sí entre concentraciones de la SN y en su interacción con fechas $(\mathrm{P} \leq 0.05)$. En la concentración de $50 \%$ de la SN se registró la mayor biomasa seca de cormo en el primer muestreo en ambas fechas de siembra, mientras que en el segundo muestreo únicamente hubo diferencias significativas en la primera fecha de siembra en donde las plantas crecidas en la solución a $100 \%$ presentaron la menor biomasa de cormo (Figura 1A y B). En el tercer muestreo se observó mayor biomasa seca de cormo en la primera fecha de siembra con $75 \%$ de la concentración de la SN (Figura $1 \mathrm{~A}$ ), y en la segunda fecha de siembra con la concentración de 25 \% (Figura1 B).

Cuadro 1. Comparación de medias del efecto de la fecha de siembra y concentración de la solución nutritiva (SN) en la altura de planta de freesia (Freesia $x$ hybrida) cultivadas en el Valle de México a la intemperie bajo malla sombra de color rojo con $15 \%$ de transmisión de la radiación solar durante el invierno 2010-2011.

\begin{tabular}{|c|c|c|c|c|c|c|c|c|c|}
\hline \multirow{3}{*}{$\mathrm{SN}(\%)$} & \multicolumn{9}{|c|}{ Altura de la planta $(\mathrm{cm})$} \\
\hline & \multicolumn{3}{|c|}{ Primer muestreo } & \multicolumn{3}{|c|}{ Segundo muestreo } & \multicolumn{3}{|c|}{ Tercer muestreo } \\
\hline & F1 & $\mathrm{F} 2$ & Media & $\mathrm{F} 1$ & $\mathrm{~F} 2$ & Media & F1 & $\mathrm{F} 2$ & Media \\
\hline 25 & $29.5 \mathrm{a}$ & $31.5 \mathrm{a}$ & $30.5 \mathrm{~A}$ & $38.6 \mathrm{a}$ & $39.8 \mathrm{a}$ & $39.2 \mathrm{~A}$ & $58.8 \mathrm{a}$ & 59.9 a & $59.4 \mathrm{~A}$ \\
\hline 50 & $31.3 \mathrm{a}$ & $28.7 \mathrm{a}$ & $30.0 \mathrm{AB}$ & $33.7 \mathrm{a}$ & $37.6 \mathrm{a}$ & $35.6 \mathrm{~A}$ & $62.6 \mathrm{a}$ & $54.0 \mathrm{~b}$ & $58.3 \mathrm{~A}$ \\
\hline 75 & $31.7 \mathrm{a}$ & $26.7 \mathrm{a}$ & $29.2 \mathrm{AB}$ & $37.2 \mathrm{a}$ & $35.8 \mathrm{a}$ & $36.5 \mathrm{~A}$ & $54.3 \mathrm{~b}$ & $47.5 \mathrm{bc}$ & $50.9 \mathrm{~B}$ \\
\hline 100 & $25.9 \mathrm{a}$ & $26.6 \mathrm{a}$ & $26.2 \mathrm{~B}$ & $35.5 \mathrm{a}$ & $35.4 \mathrm{a}$ & $35.4 \mathrm{~A}$ & $52.1 \mathrm{~b}$ & $46.0 \mathrm{c}$ & $49.0 \mathrm{~B}$ \\
\hline Media & $29.6 \mathrm{~A}$ & $28.3 \mathrm{~A}$ & & $36.2 \mathrm{~A}$ & $37.1 \mathrm{~A}$ & & $56.9 \mathrm{~A}$ & $51.8 \mathrm{~B}$ & \\
\hline
\end{tabular}

Medias con letras mayúsculas iguales en columnas o hileras de cada muestreo, son estadísticamente iguales entre sí, y letras minúsculas indican diferencias en interacción (Tukey, 0.05). F1 = primera fecha de siembra (22 de septiembre de 2010); F2 = segunda fecha de siembra (18 de octubre de 2010). 
Cuadro 2. Comparación de medias del efecto de la fecha de siembra y concentración de solución nutritiva (SN) sobre el área foliar.

\begin{tabular}{|c|c|c|c|c|c|c|c|c|c|}
\hline \multirow{3}{*}{$\mathrm{SN}(\%)$} & \multicolumn{9}{|c|}{ Área foliar $\left(\mathrm{cm}^{2}\right)$} \\
\hline & \multicolumn{3}{|c|}{ Primer muestreo } & \multicolumn{3}{|c|}{ Segundo muestreo } & \multicolumn{3}{|c|}{ Tercer muestreo } \\
\hline & F1 & $\mathrm{F} 2$ & Media & $\mathrm{F} 1$ & $\mathrm{~F} 2$ & Media & $\mathrm{F} 1$ & $\mathrm{~F} 2$ & Media \\
\hline 50 & $125.9 \mathrm{a}$ & $127.1 \mathrm{a}$ & $126.5 \mathrm{~A}$ & $178.5 \mathrm{~b}$ & $176.0 \mathrm{bc}$ & $177.2 \mathrm{~B}$ & $377.0 \mathrm{a}$ & $315.9 \mathrm{bc}$ & $346.4 \mathrm{~A}$ \\
\hline 75 & $72.3 \mathrm{bc}$ & $92.9 \mathrm{~b}$ & $82.6 \mathrm{~B}$ & $261.6 \mathrm{a}$ & $153.5 \mathrm{bc}$ & $207.5 \mathrm{~A}$ & $287.4 \mathrm{c}$ & $338.6 \mathrm{ab}$ & $313.0 \mathrm{~B}$ \\
\hline Media & $84.8 \mathrm{~A}$ & $90.3 \mathrm{~A}$ & & $184.9 \mathrm{~A}$ & $166.6 \mathrm{~B}$ & & $252.4 \mathrm{~B}$ & $271.9 \mathrm{~A}$ & \\
\hline
\end{tabular}

Medias con letras mayúsculas iguales en columnas o hileras de cada muestreo, son estadísticamente iguales entre sí, y letras minúsculas indican diferencias en interacción (Tukey, 0.05). F1 = primera fecha de siembra (22 de septiembre de 2010); F2 = segunda fecha de siembra (18 de octubre de 2010 ).

En el primero y segundo muestreo la acumulación de biomasa seca en tallo, raíz y hoja fue igual entre tratamientos, mientras que en el tercer muestreo las plantas crecidas con $100 \%$ de la solución Steiner acumularon la menor cantidad de biomasa en tallo y hoja (Figura 1 C, D, E, F, G y H). En la biomasa seca de raíz no se observaron diferencias entre tratamientos. En el tercer muestreo la menor biomasa seca total se obtuvo con $100 \%$ de la SN de Steiner.

\section{Fluorescencia inducida en la hoja}

La fluorescencia inicial (F0) fue igual en todas las concentraciones de la SN aplicadas en ambas fechas de medición (Cuadros 3 y 4). En la fluorescencia máxima (Fm) se encontraron diferencias significativas entre soluciones nutritivas en la segunda fecha de medición (Cuadro 4), donde la concentración de la SN a $75 \%$ obtuvo el menor valor. En la interacción concentración de $\mathrm{SN} x$ fecha de muestreo, los valores mayor y menor de Fm fueron obtenidos con las concentraciones de 50 y $75 \%$, respectivamente.

Sólo en la primera fecha de medición (Cuadros 3 y 4) se encontraron diferencias estadísticas $(\mathrm{P} \leq 0.05)$ entre fechas de siembra en la fluorescencia variable $(\mathrm{Fv})$, pero no entre concentraciones de la SN. En la interacción, la menor Fv se obtuvo en la primera, y el mayor en la segunda fecha, ambas con la concentración de $50 \%$. La eficiencia cuántica máxima del fotosistema II ( Fm/Fv) varió de 0.80 a 0.83 , pero fue igual entre tratamientos $(\mathrm{P}>0.05)$ en ambas fechas de medición (Cuadros 3 y 4 ).

\section{Lecturas SPAD}

Las lecturas SPAD en las hojas de freesia presentaron diferencias estadísticas entre tratamientos $(\mathrm{P} \leq 0.05)$. Las plantas desarrolladas con SN a $25 \%$ presentaron los valores más altos en comparación con las que crecieron en SN a $100 \%$ (Cuadro 5). Entre fechas de plantación no se observaron diferencias significativas $(\mathrm{P}>0.05)$. La mejor interacción correspondió a la concentración de $25 \%$ en la segunda fecha de siembra.

\section{Tallo floral}

El corte de tallos florales se efectuó a los 149 DDS en la primera fecha de siembra y a los 141 DDS en la segunda fecha. La concentración de la SN, las fechas de siembra y su interacción tuvieron efectos significativos $(\mathrm{P} \leq 0.05)$ en la longitud del tallo floral. La mayor longitud de tallo floral se obtuvo con las SN de 25 y $50 \%$, con valores de 59.6 y 61.6 $\mathrm{cm}$ de longitud media (Figura 2), mientras que la menor fue con la SN de $100 \%$ que tuvo cerca de 1/3 menos altura que con la SN de $25 \%$. Entre fechas de siembra no hubo diferencias significativas $(\mathrm{P}>0.05)$.

Las concentraciones de la SN y fechas de siembra, así como su interacción, tuvieron efectos significativos en longitud de la espiga, número de flores por espiga y grosor del tallo (Cuadro 6). Con la concentración de $25 \%$ se obtuvieron los mayores valores en longitud de espiga, número de flores por espiga y grosor de tallo, en ambas fechas de siembra. La menor longitud de espiga en la primera fecha y menor grosor de tallo en la segunda fecha fueron obtenidos con el tratamiento de SN $100 \%$. No se observaron diferencias significativas entre tratamientos $(\mathrm{P}>0.05)$ en el peso de la biomasa fresca de tallos florales. Los tallos florales con tratamiento a concentración de $25 \%$ tuvieron mayor vida en florero ( $14 \mathrm{~d}$ ) debido a su mayor número de flores. El resto de los tratamientos duraron un promedio de $11 \mathrm{~d}$. Los botones florales formados en la espiga abrieron en todos los tratamientos. 

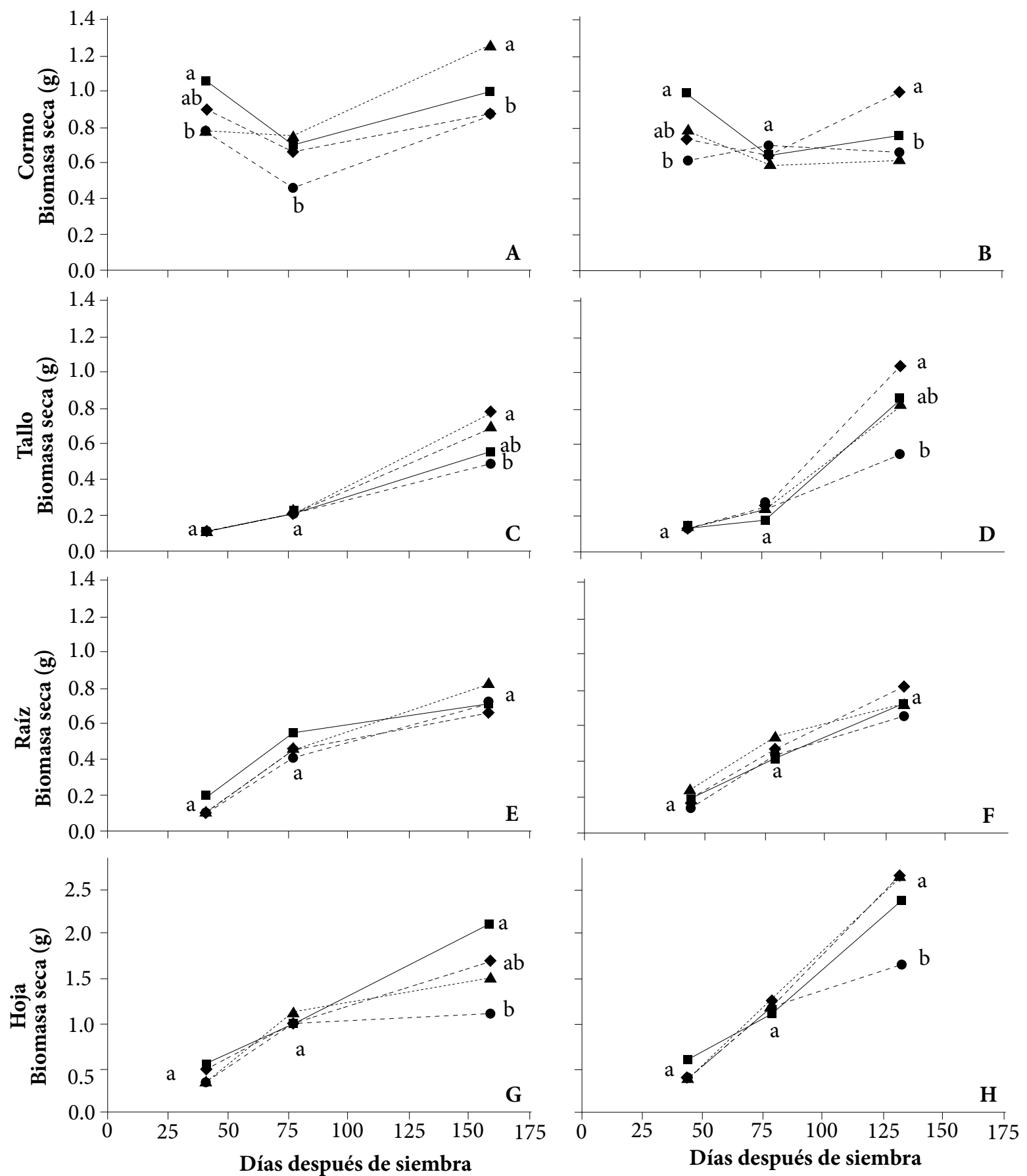

Figura 1. Biomasa seca por órgano vegetal durante el desarrollo de Freesia $x$ hybrida cultivada a concentraciones de $100 \%$ ( - •-), $75 \%(\ldots), 50 \%\left(\_\right)$y $25 \%(-\ldots)$ de solución nutritiva de Steiner, plantadas el 22 de septiembre 2010 (A, C, E y G) y el 18 de octubre 2010 (B, D, F y H) en el Valle de México. Letras minúsculas diferentes por fecha de muestreo indican diferencias estadísticas (Tukey, 0.05). 
Cuadro 3. Comparación de medias del efecto de la fecha de siembra y concentración de solución nutritiva (SN) en fluorescencia inducida (13 de diciembre 2010).

\begin{tabular}{|c|c|c|c|c|c|c|}
\hline \multirow{3}{*}{ SN (\%) } & \multicolumn{6}{|c|}{ Fluorescencia inducida } \\
\hline & \multicolumn{3}{|c|}{ F0 (fluorescencia inicial) } & \multicolumn{3}{|c|}{ Fm (fluorescencia máxima) } \\
\hline & F1 & F2 & Media & F1 & F2 & Media \\
\hline 25 & $481 \mathrm{a}$ & $488 \mathrm{a}$ & $484 \mathrm{~A}$ & $2834 \mathrm{a}$ & $2900 \mathrm{a}$ & $2867 \mathrm{~A}$ \\
\hline 50 & $442 \mathrm{a}$ & $481 \mathrm{a}$ & $462 \mathrm{~A}$ & $2253 \mathrm{a}$ & $2901 \mathrm{a}$ & $2577 \mathrm{~A}$ \\
\hline 75 & $438 \mathrm{a}$ & $444 \mathrm{a}$ & $441 \mathrm{~A}$ & $2562 \mathrm{a}$ & $2397 \mathrm{a}$ & $2480 \mathrm{~A}$ \\
\hline 100 & $494 \mathrm{a}$ & 438 a & $461 \mathrm{~A}$ & $2554 \mathrm{a}$ & $2770 \mathrm{a}$ & $2662 \mathrm{~A}$ \\
\hline Media & $463 \mathrm{~A}$ & $463 \mathrm{~A}$ & & $2551 \mathrm{~A}$ & $2742 \mathrm{~A}$ & \\
\hline \multirow{2}{*}{ SN (\%) } & \multicolumn{3}{|c|}{ Fv (fluroescencia variable) } & \multicolumn{3}{|c|}{$\mathrm{Fm} / \mathrm{Fv}$} \\
\hline & $\mathrm{F} 1$ & $\mathrm{~F} 2$ & Media & $\mathrm{F} 1$ & $\mathrm{~F} 2$ & Media \\
\hline 25 & $2353 \mathrm{a}$ & $2412 \mathrm{a}$ & $2382 \mathrm{~A}$ & $0.83 \mathrm{a}$ & $0.83 \mathrm{a}$ & $0.83 \mathrm{~A}$ \\
\hline 50 & $1811 \mathrm{~b}$ & $2419 \mathrm{a}$ & $2189 \mathrm{~A}$ & $0.80 \mathrm{a}$ & $0.83 \mathrm{a}$ & $0.81 \mathrm{~A}$ \\
\hline 75 & $2124 \mathrm{ab}$ & $2253 \mathrm{ab}$ & $2115 \mathrm{~A}$ & $0.82 \mathrm{a}$ & $0.81 \mathrm{a}$ & $0.82 \mathrm{~A}$ \\
\hline 100 & $2260 \mathrm{ab}$ & $2342 \mathrm{a}$ & $2301 \mathrm{~A}$ & $0.82 \mathrm{a}$ & $0.80 \mathrm{a}$ & $0.81 \mathrm{~A}$ \\
\hline Media & $2137 \mathrm{~B}$ & $2357 \mathrm{~A}$ & & $0.82 \mathrm{~A}$ & $0.82 \mathrm{~A}$ & \\
\hline
\end{tabular}

Medias con letras mayúsculas iguales en columnas o hileras de cada muestreo, son estadísticamente iguales entre sí, y letras minúsculas indican diferencias en interacción (Tukey, 0.05). F1 = primera fecha de siembra (22 de septiembre de 2010); F2 = segunda fecha de siembra (18 de octubre de 2010$)$.

Cuadro 4. Comparación de medias del efecto de la fecha de siembra y concentración de solución nutritiva (SN) en fluorescencia inducida (25 de enero de 2011 ).

\begin{tabular}{|c|c|c|c|c|c|c|}
\hline \multirow{3}{*}{$\mathrm{SN}(\%)$} & \multicolumn{6}{|c|}{ Fluorescencia inducida } \\
\hline & \multicolumn{3}{|c|}{ F0 (flourescencia inicial) } & \multicolumn{3}{|c|}{ Fm (fluorescencia máxima) } \\
\hline & $\mathrm{F} 1$ & $\mathrm{~F} 2$ & Media & $\mathrm{F} 1$ & $\mathrm{~F} 2$ & Media \\
\hline 25 & $478 \mathrm{a}$ & $491 \mathrm{a}$ & $485 \mathrm{~A}$ & $2951 \mathrm{ab}$ & $3002 \mathrm{ab}$ & $2976 \mathrm{~A}$ \\
\hline 50 & $484 \mathrm{a}$ & $513 \mathrm{a}$ & $498 \mathrm{~A}$ & $2863 \mathrm{ab}$ & $3097 \mathrm{a}$ & $2980 \mathrm{~A}$ \\
\hline 75 & $436 \mathrm{a}$ & $473 \mathrm{a}$ & $454 \mathrm{~A}$ & $2636 \mathrm{ab}$ & $2537 \mathrm{~b}$ & $2586 \mathrm{~B}$ \\
\hline 100 & $469 \mathrm{a}$ & $490 \mathrm{a}$ & $479 \mathrm{~A}$ & $2694 \mathrm{ab}$ & $2955 \mathrm{ab}$ & $2825 \mathrm{AB}$ \\
\hline Media & $467 \mathrm{~A}$ & $492 \mathrm{~A}$ & & $2786 \mathrm{~A}$ & $2898 \mathrm{~A}$ & \\
\hline \multirow{2}{*}{$\mathrm{SN}(\%)$} & \multicolumn{3}{|c|}{ Fv (flourescencia variable) } & \multicolumn{3}{|c|}{$\mathrm{Fm} / \mathrm{Fv}$} \\
\hline & F1 & $\mathrm{F} 2$ & Media & $\mathrm{F} 1$ & $\mathrm{~F} 2$ & Media \\
\hline 25 & $2460 \mathrm{a}$ & $2441 \mathrm{a}$ & $2450 \mathrm{~A}$ & $0.82 \mathrm{a}$ & $0.83 \mathrm{a}$ & $0.83 \mathrm{~A}$ \\
\hline 50 & 2379 a & $2594 \mathrm{a}$ & 2487 A & $0.83 \mathrm{a}$ & $0.83 \mathrm{a}$ & $0.83 \mathrm{~A}$ \\
\hline 75 & $2218 \mathrm{a}$ & $2306 \mathrm{a}$ & $2262 \mathrm{~A}$ & $0.82 \mathrm{a}$ & $0.81 \mathrm{a}$ & $0.82 \mathrm{~A}$ \\
\hline 100 & 2199 a & $2345 \mathrm{a}$ & $2272 \mathrm{~A}$ & $0.81 \mathrm{a}$ & $0.81 \mathrm{a}$ & $0.81 \mathrm{~A}$ \\
\hline Media & $2314 \mathrm{~A}$ & $2422 \mathrm{~A}$ & & $0.82 \mathrm{~A}$ & $0.82 \mathrm{~A}$ & \\
\hline
\end{tabular}

Medias con letras mayúsculas iguales en columnas o hileras de cada muestreo, son estadísticamente iguales entre sí, y letras minúsculas indican diferencias en interacción (Tukey, 0.05). F1 = primera fecha de siembra (22 de septiembre de 2010); F2 = segunda fecha de siembra (18 de octubre de 2010). 
Cuadro 5. Comparación de medias del efecto de la fecha de siembra y concentración de solución nutritiva (SN) en lecturas SPAD.

\begin{tabular}{|c|c|c|c|}
\hline \multirow{2}{*}{$\mathrm{SN}(\%)$} & \multicolumn{3}{|c|}{ Lecturas SPAD } \\
\hline & $\mathrm{F} 1$ & $\mathrm{~F} 2$ & Media \\
\hline 25 & $70.5 \mathrm{ab}$ & $72.9 \mathrm{a}$ & $71.7 \mathrm{~A}$ \\
\hline 50 & $68.5 \mathrm{ab}$ & $66.0 \mathrm{~b}$ & $67.3 \mathrm{~B}$ \\
\hline 75 & $67.5 \mathrm{ab}$ & $69.0 \mathrm{ab}$ & $68.2 \mathrm{~B}$ \\
\hline 100 & $60.0 \mathrm{c}$ & $65.4 \mathrm{bc}$ & $62.7 \mathrm{C}$ \\
\hline Media & $67.1 \mathrm{~A}$ & $68.0 \mathrm{~A}$ & \\
\hline
\end{tabular}

Medias con letras mayúsculas iguales en columnas o hileras de cada muestreo, son estadísticamente iguales entre sí, y letras minúsculas indican diferencias en interacción (Tukey, 0.05). F1 = primera fecha de siembra (22 de septiembre de 2010); F2 = segunda fecha de siembra (18 de octubre de 2010).

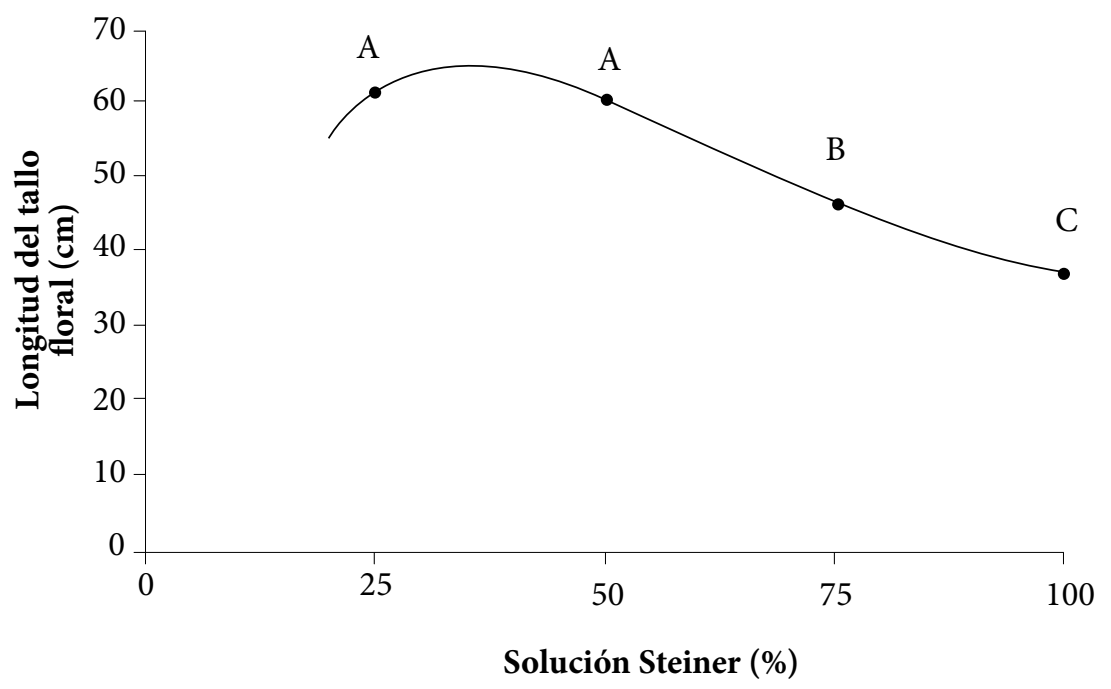

Figura 2. Concentración de la solución nutritiva Steiner y la longitud de tallo floral $(\mathrm{cm})$ de freesia crecida durante el invierno 2010-2011. Letras diferentes por fecha de muestreo indican diferencias estadísticas (Tukey, 0.05). Los puntos representan promedios de dos fechas de siembra, y la línea continua es la tendencia del modelo Steinhart-Hart. $y=1 /(9.68 E-02-3.45 E-02$ $\left.x \ln (x)+9.12 E-04 x \ln (x)^{3}\right)\left(\mathrm{n}=4 ; \mathrm{R}^{2}=0.99\right)$, donde: " $x$ " es la concentración (\%) de la solución nutritiva de Steiner, $\mathrm{y}$ " $y$ " es longitud del tallo floral $(\mathrm{cm})$.

Cuadro 6. Comparación de medias de efecto de la concentración de solución nutritiva (SN) y fechas de siembra en variables de calidad del tallo floral.

\begin{tabular}{|c|c|c|c|c|c|c|}
\hline \multirow{2}{*}{ SN (\%) } & \multicolumn{2}{|c|}{ Longitud de espiga $(\mathrm{cm})$} & \multicolumn{2}{|c|}{ Número de flores por espiga } & \multicolumn{2}{|c|}{ Grosor de tallo (mm) } \\
\hline & F1 & F2 & F1 & F2 & F1 & F2 \\
\hline 25 & $10.8 \mathrm{a}$ & $11.1 \mathrm{a}$ & $11 \mathrm{a}$ & $12 \mathrm{a}$ & $4.9 \mathrm{a}$ & $5.1 \mathrm{a}$ \\
\hline 50 & $9.6 \mathrm{~b}$ & $9.5 \mathrm{~b}$ & $9 \mathrm{~b}$ & $9 \mathrm{~b}$ & $4.7 \mathrm{a}$ & $4.9 \mathrm{a}$ \\
\hline 75 & $9.3 \mathrm{~b}$ & $9.3 \mathrm{~b}$ & $8 \mathrm{~b}$ & $9 \mathrm{~b}$ & $4.5 \mathrm{a}$ & $4.3 \mathrm{~b}$ \\
\hline 100 & $8.5 \mathrm{c}$ & $8.5 \mathrm{~b}$ & $8 \mathrm{~b}$ & $8 \mathrm{~b}$ & $3.4 \mathrm{~b}$ & $3.6 \mathrm{c}$ \\
\hline
\end{tabular}

Medias con letras iguales no son estadísticamente diferentes (Tukey, 0.05). F1 = primera fecha de siembra (22 de septiembre de 2010); F2 = segunda fecha de siembra (18 de octubre de 2010). 


\section{DISCUSIÓN}

En esta investigación se detectó una notable disminución en altura de planta, longitud del tallo floral, área foliar, menor concentración de clorofila, longitud de espiga y grosor de tallo con la solución Steiner a $100 \%$. En contraste, la concentración de $25 \%$ produjo mayores valores de altura de planta, contenido de clorofila y calidad del tallo floral, esta última en términos de longitud del tallo floral, longitud de espiga, número de flores por espiga, grosor de tallo y número de días de vida de florero. Los valores obtenidos en longitud de tallo y número de flores para Freesia $x$ hybrida con 25 y 50 \% de la solución Steiner, con tallos de más de 50 $\mathrm{cm}$ y más de ocho flores por espiga, superaron a la calidad extra descrita en las normas de calidad para flor cortada (Medio Ambiente, Agricultura y Agua, 1998). En cambio, con 75 y 100 \% de la solución Steiner sólo fue posible cumplir con el número de flores mínimo para esta categoría.

La máxima longitud de tallo se obtuvo con las concentraciones de 25 y $50 \%$ de la solución Steiner. Con el modelo Steinhart-Hart (Figura 2) se pudo predecir que la longitud máxima de $65.3 \mathrm{~cm}$ del tallo floral sería con $34.7 \%$ de la solución Steiner. El modelo también predijo una caída de la longitud del tallo floral más pronunciada cuando disminuye la concentración de la solución nutritiva por debajo de 34.7 \% de la solución Steiner que cuando aumenta sobre este valor.

La amplia respuesta de freesia a diferentes concentraciones de nutrimentos fue reportado antes por Smith y Danks (1985). El exceso de nutrientes disminuye el potencial osmótico del sustrato por altos niveles de sales y esto aumenta la conductividad eléctrica (CE). A diferencia de lo que ocurre en el suelo donde la salinidad es causada por una mezcla de iones inorgánicos, en hidroponía la salinidad es causada por los nutrimentos añadidos, que en los suelos de invernadero holandeses un tercio de los iones corresponden de $\mathrm{Ny}$ K (Sonneveld et al., 2004).

El exceso de sales nutritivas ocasiona daños en la raíz y en el crecimiento del cultivo, como ocurre en gerbera (Gerbera hybrida) al reciclar la solución nutritiva drenada cuya $\mathrm{CE}$ es alta (Savvas y Gizas, 2002), así como en gladiolo (Gladiolus grandiflorus Hort) (Haouala y Salhi, 2011) y en freesia cuando son irrigadas con agua con alto contenido de sales, que causan disminución en rendimiento de flores, longitud de la espiga, así como en longitud del tallo floral, diámetro y número de flores por planta (Aydinsakir et al., 2010), similar a lo obtenido en nuestra investigación.

En la solución Steiner a 100 \% de concentración la CE fue de $2.6 \mathrm{dS} \mathrm{m}^{-1}$, que excede el rango óptimo de 1.5 a 2.0 dS $\mathrm{m}^{-1}$ para el crecimiento de la freesia según Larson (1988), pero no al umbral máximo de $2.4 \mathrm{dS} \mathrm{m}^{-1}$ que propusieron Sonneveld et al. (2004). Por sus consecuencias observadas en la presente investigación en el crecimiento y calidad del tallo floral, se puede considerar que esta concentración de la SN no es recomendable para freesia. Sin embargo, la eficiencia cuántica máxima del fotosistema II (Fm/Fv) fue igual entre las concentraciones evaluadas de la $\mathrm{SN}$, por lo que en este contexto se infiere que las reacciones fotoquímicas de estas plantas no estuvieron bajo estrés con la concentración máxima.

Como nutrimento, el fósforo contribuye en la persistencia de la espiga floral y días a floración de freesia (Khan et al., 2012), y la deficiencia de fósforo y nitrógeno producen tallos cortos en freesia (Kidsadawanich y Ruamrungsri, 2005) y Spartina foliosa (Trnka y Zedler, 2000). La falta de uno de los tres más importantes elementos, como son nitrógeno, fósforo o potasio, puede reducir las concentraciones de los otros elementos en los tejidos, como se ha reportado en freesia (Kidsadawanich y Ruamrungsri, 2005) y en Lilium longiflorum 'Nellie White' (Niedziela et al., 2008). Sin embargo, esto depende de la concentración del elemento limitante como lo reportaron Verlinden y MacDonald (2007) quienes en plantas de Limonium encontraron que las concentraciones de $\mathrm{P}$ de 0 a $60 \mathrm{mg} \mathrm{L}^{-1}$ alteraron significativamente la longitud del tallo, mientras que las concentraciones de $\mathrm{N}$ de 100 a $400 \mathrm{mg} \mathrm{L}^{-1}$ no tuvieron efecto.

En cambio, en la presente investigación se observó que con 100 y $25 \%$ de la solución Steiner se obtuvieron las menores y mayores lecturas SPAD, respectivamente. Ésta es una medida indirecta de la concentración de clorofila y en consecuencia de la cantidad de nitrógeno en las hojas. Se puede considerar entonces que el menor potencial osmótico que debió ocurrir en la zona radical con la dosis completa de la solución Steiner, disminuyó la absorción de $\mathrm{N}$ por la planta, así como la de $\mathrm{P}$ y otros elementos nutritivos. Estos resultados indican que el cultivo no requiere altas concentraciones nutrimentales, lo que es una ventaja para bajar costos de producción.

Freesia es considerada como una planta ornamental que aún está en proceso de domesticación, y con una dosis de $100 \mathrm{~N}: 50 \mathrm{P}: 200 \mathrm{~K} \mathrm{mg} \mathrm{L}^{-1}$ es suficiente para producirla en un sustrato compuesto de arena, cascarilla de arroz y fibra de coco (Ruamrungsri et al., 2011). Con la solución Steiner a $100 \%$ se aplicó más nitrógeno $\left(168 \mathrm{mg} \mathrm{L}^{-1}\right)$ y potasio $(273$ $\mathrm{mg} \mathrm{L}^{-1}$ ) pero menos fósforo $\left(31 \mathrm{mg} \mathrm{L}^{-1}\right)$ que la recomendada por estos autores. Sin embargo, con $1 / 4$ de esta cantidad, es decir con $42 \mathrm{~N}: 8 \mathrm{P}: 68 \mathrm{~K} \mathrm{mg} \mathrm{L}^{-1}$, se pueden producir freesias de calidad a partir de cormos de $2.5 \mathrm{~cm}$ de diámetro crecidos bajo malla sombra roja con $15 \%$ de transmisión de la radiación solar, lo cual concuerda con Thomas et al. (1998) quienes indicaron que la freesia es una especie 
que demanda bajos niveles de nutrientes. Al respecto, los cormos funcionan como una fuente de carbohidratos y nutrientes para el crecimiento de la planta de freesia, porque a partir de cormos de menor tamaño las plantas responden favorablemente al aumentar la dosis de fertilizante (Żurawik y Placek, 2011).

\section{CONCLUSIONES}

El mejor crecimiento y calidad del tallo floral de Freesia $x$ hybrida cultivada en un sistema hidropónico bajo malla sombra roja de $15 \%$ de transmisión de la radiación solar durante el invierno en el Valle de México, se obtuvo con solución Steiner a $25 \%$ de su concentración.

\section{BIBLIOGRAFÍA}

ASERCA, Apoyos y Servicios a la Comercialización Agropecuaria (2006) La floricultura mexicana, el gigante que está despertando. Claridades Agropecuarias 154:3-38.

Aydinsakir K., A. Tepe and D. Buyuktas (2010) Effects of saline irrigation water applications on quality characteristics of Freesia grown in greenhouse. Akdeniz Universitesi Ziraat Fakültesi Dergisi 23:41-46.

Bullock D. G. and D. S. Anderson (1998) Evaluation of the Minolta SPAD-502 chlorophyll meter for nitrogen management in corn. Journal of Plant Nutrition 21:741-755.

Carpena O., A. M. Rodríguez y M. J. Sarro (1987) Evaluación de los contenidos minerales de raíz, tallo y hoja de las plantas como índice de nutrición. Anales de Edafologia y Agrobiologia 46:117-127.

Dole J. and H. Wilkins (1999) Floriculture. Principles and Species. New Jersey, Prentice-Hall. 613 p.

Hansatech Instruments Ltd. (2006) Operations Manual. Hansatech Instruments Ltd. King's Lynn, Norfolk, England. 85 p.

Haouala F. and I. Salhi (2011) Effets du NaClsur la croissance, la flroaisonet la bulbaison du glaïeul (Gladiolus grandiflorus Hort). Revue Suisse de Viticulture, Arboriculture, Horticulture 43:378383.

Khan M. K., M. Sajid, A. Rab, I. Jan, H. Zada, M. Zamin, I. Haq, A. Zaman, S. T. Shah and A. U. Rehman (2012) Influence of nitro- gen and phosphorus on flower and corm production of Freesia. African Journal of Biotechnology 11:11936-11942.

Kidsadawanich H. and S. Ruamrungsri (2005) Nitrogen, phosphorus and potassium deficiency in Freesia spp. J. Agric. 21:197-204.

Lara H. A. (1999) Manejo de la solución nutritiva en la producción de tomate en hidroponía. Terra Latinoamericana 17:221-229.

Larson R. A. (1988) Introducción a la floricultura. la ed. AGT Editor, S.A. 551 p.

Medio Ambiente, Agricultura y Agua (1998) Normas de calidad para flor cortada. Murcia (España), Consejería de Medio Ambiente, Agricultura y Agua. 50 p. (Información $\mathrm{N}^{\circ} 55$ ).

Niedziela C. E., S. H Kim, P. V. Nelson and A. A. de Hertogh (2008) Effects of N-P-K deficiency and temperature regime on the growth and development of Lilium longilforum 'Nellie White' during bulb production under phytotron conditions. Scientia Horticulturae 116:430-436.

Rees A. (1992) Ornamental Bulbs, Corms and Tubers. Wallingfor. CAB International. $220 \mathrm{p}$.

Ruamrungsri S., W. Bundithya, N. Potapohn, N. Ohtake, K. Sueyoshi and T. Ohyama (2011) Effect of NPK levels on growth and bulb quality of some geophytes in substrate culture. Acta Horticulturae 886:213-218.

SAS Institute (2010) SAS/STAT User's guide. Ver. 9.2 SAS Institute, Inc. Cary, North Carolina, U.S.A. 1689 p.

Savvas D., G. Gizas (2002) Response of hydroponically grown gerbera to nutrient solution recycling and different nutrient cation ratios. Scientia Horticulturae 96:267-280.

Smith D. and P. N. Danks (1985) Freesias. Gower Books. London. 45 p.

Sonneveld C., A. L. van den Bos and W. Voogt (2004) Modeling osmotic salinity effects on yield characteristics of substrate-grown greenhouse crops. Journal of Plant Nutrrition 27:1931-1951.

Steiner A. A. (1961) A universal method for preparing nutrient solution of a certain desired composition. Plant Soil 15:134-154.

Thomas M., S. Matheson and M. Spurwaya (1998) Nutrition of container-grown freesias. Journal of Plant Nutrrition 21:2485-2496.

Trnka S. and J. B. Zedler (2000) Site conditions, not parental phenotype, determine the height of Spartina foliosa. Estuaries 23:572-582.

Verlinden S. and L. McDonald (2007) Productivity and quality of statice (Limonium sinuatum cv. Soiree Mix) and cockscomb (Celosia argentea $\mathrm{cv}$. Chief Mix) under organic and inorganic fertilization regiments. Scientia Horticulturae 114:199-206.

Wang L. (2007) Freesia. Freesia $x$ hybrida. In: Flower Breeding and Genetics. N O Anderson. (ed). Springer. Netherlands. pp:665-693.

Żurawik P. and M. Placek (2011) The influence of fertilization on quality of inflorescence of easy pot Freesia (Freesia Eckl. ex Klatt) grown form adventitious corms. Acta Agrobotanica 64:59-66. 